\title{
Desafios da Política Nacional de Atenção Básica à Saúde
}

\author{
Challenges of the National Primary Health Care Policy
}

\section{Desafíos de la Política Nacional de Atención Primaria de Salud}

\section{Guilherme Carraro Ganem Vieira da Silva ${ }^{1 *}$, Marcos Alex Mendes da Silva ${ }^{2}$, Patrícia Pereira Nogueira $^{3}$, Oswaldo Luiz Cecílio Barbosa ${ }^{4}$}

Como citar esse artigo. da Silva, GCGV; da Silva, MAM; Nogueira, PP; Barbosa, OLC. Desafios da Política Nacional de Atenção Básica à Saúde. Revista Pró-UniverSUS. 2021 Jan./Jun.; 12 (1): $60-65$

\section{Resumo}

Trata-se de análise documental de duas portarias ministeriais, que dispõem sobre a Política Nacional de Atenção Básica, publicadas em 2012 e 2017. A atenção primaria à saúde (APS) é estruturada, a nível mundial, desde o século XX, criando o conceito de rede territorial de serviços nucleada a partir de centros primários, com autoridade regional. O Sistema único de Saúde (SUS) brasileiro determina o dever do Estado em fornecer cuidados e assistência para todo cidadão. Inicialmente, o modelo de APS no Brasil passou a funcionar por meio do Programa saúde da família (PSF) e do programa de agentes comunitários de saúde (PACS). Destacam-se os princípios de acolhimento, vínculo, territorialização, trabalho em equipe multiprofissional, ações coletivas com o compromisso de reorganizar prática de atenção à saúde, estímulo à construção de uma consciência participativa e reforço às linhas do cuidado em saúde. A saúde pública no Brasil teve avanços extremos ao longo dos anos e o aumento de investimento possibilitou a ampliação do acesso à saúde e uma atenção integral, garantida pela implementação da política nacional de atenção básica em 2006, que posteriormente foi reformulada, em 2012 e em 2017. A PNAB de 2017 trouxe mudanças muito significativas e com possíveis efeitos sobre a integralidade do sistema de saúde, que antes considerava a ESF como opção preferencial de cuidado, contando com equipes completas e carga horária de trabalho visando à longitudinalidade, passou a aceitar outras formas de equipes de trabalho, com o número de profissionais e suas cargas horárias de trabalho reduzidas.

Palavras-chave: Saúde Pública; Atenção Básica; Programa de Saúde da Família; Política Nacional de Atenção Básica em Saúde; Desmonte do SUS.

\begin{abstract}
This is a documental analysis of two ministerial ordinances, which provide for the National Primary Care Policy, published in 2012 and 2017. Primary health care (PHC) has been structured worldwide since the 20th century, creating the concept of a territorial network of nucleated services from primary centers with regional authority. The Brazilian Unified Health System (SUS) determines the state's duty to provide care and assistance to every citizen. Initially, the PHC model in Brazil began to function through the Family Health Program (FHP) and the Community Health Agents Program (PACS). The principles of welcoming, bonding, territorialization, multiprofessional teamwork, collective actions with the commitment to reorganize health care practice, encouraging the construction of a participatory awareness and strengthening the lines of health care stand out. Public health in Brazil has made extreme advances over the years and the increase in investment has enabled the expansion of access to health and comprehensive care, guaranteed by the implementation of the national primary care policy in 2006, which was subsequently reformulated in 2012 and 2017. The 2017 PNAB brought very significant changes and with possible effects on the integrality of the health system, which previously considered the ESF as the preferred option of care, with complete teams and workload aimed at longitudinality, began to accept other forms of work teams, with the number of professionals and their workloads reduced.
\end{abstract}

Keywords: Public Health; Primary Care; Family Health Program; National Health Care Policy; Dismantling of the SUS.

Afiliação dos autores:

${ }^{1 *}$ Acadêmico no Curso de Odontologia, Universidade de Vassouras. Vassouras, RJ, Brasil. E-mail: guilherme.carraro96@yahoo.com.br ORCID: https://orcid.org/0000-0002-61146930

${ }^{2}$ Doutor. Docente do Curso de Odontologia, Universidade de Vassouras. Vassouras, RJ, Brasil. E-mail: marcosalexmendes@uol.com.br ORCID: https://orcid.org/0000-0003-4059$925 \mathrm{X}$

${ }^{3}$ Mestre. Docente do Curso de Odontologia, Universidade de Vassouras. Vassouras, RJ, Brasil. E-mail: patyppn193@gmail.com ORCID: https://orcid.org/0000-0003-1967-8842

${ }^{4}$ Docente do Curso de Odontologia, Universidade de Vassouras. Vassouras, RJ, Brasil. E-mail: oswaldolcbarbosa@hotmail.com ORCID: https://orcid.org/0000-0002-7668-3755 


\section{Resumen}

Se trata de un análisis documental de dos ordenanzas ministeriales, que prevén la Política Nacional de Atención Primaria, publicada en 2012 y 2017. La atención primaria de salud (PHC) se ha estructurado en todo el mundo desde el siglo XX, creando el concepto de una red territorial de servicios nucleados de centros primarios con autoridad regional. El Sistema Unificado de Salud (SUS) brasileño determina el deber del Estado de brindar atención y asistencia a todos los ciudadanos. Inicialmente, el modelo de PHC en Brasil comenzó a funcionar a través del Programa de Salud Familiar (FHP) y el Programa de Agentes de Salud Comunitaria (PACS). Destacan los principios de acogida, vinculación, territorialización, trabajo en equipo multiprofesional, acciones colectivas con el compromiso de reorganizar la práctica de la atención de la salud, fomentar la construcción de una conciencia participativa y fortalecer las líneas de la atención sanitaria. La salud pública en Brasil ha hecho avances extremos a lo largo de los años y el aumento de la inversión ha permitido la expansión del acceso a la salud y la atención integral, garantizada por la aplicación de la política nacional de atención primaria en 2006, que posteriormente fue reformulada en 2012 y 2017. El PNAB 2017 trajo cambios muy significativos y con posibles efectos en la integralidad del sistema de salud, que anteriormente consideraba el FSE como la opción preferida de atención, con equipos completos y carga de trabajo dirigida a longitudinalidad, comenzó a aceptar otras formas de equipos de trabajo, con el número de profesionales y sus cargas de trabajo reducidos.

Palabras clave: Salud Pública; Atención Primaria; Programa de Salud Familiar; Política Nacional de Atención Médica; Desmantelamiento del SUS

\section{Introdução}

A atenção primaria à saúde (APS) é estruturada, a nível mundial, desde o século XX, através do relatório de Dawson de $1922^{1}$, criando o conceito de rede territorial de serviços nucleada a partir de centros primários, com autoridade regional. O relatório de Dawson serviu de base para criação do sistema de saúde inglês ${ }^{1}$ e dos demais derivados.

Na década de 1970, foi realizada a conferência internacional de Alma Ata, influenciada pelo contexto político e econômico mundial e pelos custos destinados ao setor de saúde, que propunha cuidados primários em saúde como foco ${ }^{1}$. Diferentes países no mundo possuem APS em seu sistema de saúde e utilizam a conferência como referência para suas ações ${ }^{1}$.

O Sistema único de Saúde (SUS) brasileiro foi criado em 1988 pela Constituição Federal ${ }^{1}$ e determina o dever do Estado em fornecer cuidados e assistência para todo cidadão. Em 1990, foi aprovada pelo Congresso Nacional a Lei Orgânica da Saúde, que detalhava os meios e as estratégias para sua operacionalização ${ }^{1}$.

Inicialmente, o modelo de APS no Brasil passou a funcionar por meio do Programa saúde da família (PSF) e do programa de agentes comunitários de saúde (PACS) $^{2}$. Em 1996, substituiu-se o termo PSF por ESF (Estratégia saúde da família) atribuindo um caráter contínuo ao programa, que consiste em um sistema em que integra PSF e PACS, e visa à promoção de saúde ${ }^{2}$. Destacam-se os princípios de acolhimento, vínculo, territorialização, trabalho em equipe multiprofissional, ações coletivas com o compromisso de reorganizar prática de atenção à saúde, estímulo à construção de uma consciência participativa e reforço às linhas do cuidado em saúde ${ }^{1}$.

A saúde pública no Brasil teve avanços extremos ao longo dos anos e o aumento de investimento possibilitou a ampliação do acesso à saúde e uma atenção integral, garantida pela implementação da política nacional de atenção básica em 2006, que posteriormente foi reformulada, em 2012 e em 2017. Em todos os documentos orientadores, a atenção básica de saúde (ABS) aparece como sinônimo de Atenção Primária à Saúde (APS) e corresponde ao conjunto de ações no âmbito individual e coletivo, que envolvem a promoção de saúde, tratamentos, diagnósticos, prevenção de agravos, redução de danos e manutenção da saúde, visando fortalecer a ação integral e com longevidade.

Nacionalmente, a APS é porta de entrada para os usuários do SUS e se organiza de acordo com o mesmo. Visa a fornecer acesso universal e contínuo aos demais níveis de complexidade dos serviços de saúde, garantido pelos princípios da universalidade, da integralidade e da equidade, reafirmando a abrangência do sistema. Os anos que se seguiram após a implementação do SUS acompanharam a organização da APS por meio de diferentes iniciativas ministeriais, que destacavam a ESF como recurso prioritário para a sua efetivação.

Entretanto, a portaria ministerial de 2017 aponta novidades nessa priorização, tornando a ESF um elemento coadjuvante no cenário da $\mathrm{ABS}$, que passa a aceitar as equipes de atenção básica (EAB) como estratégia de funcionamento desse nível do sistema de saúde, e não somente a $\mathrm{ESF}^{3}$. Neste contexto de reversão e mudança na organização da ABS, justificase realizar uma revisão da literatura disponível para melhor compreensão dos efeitos sobre a organização da ABS no Brasil.

O objetivo deste estudo consiste em conhecer e refletir as mudanças na organização da ABS no Brasil, a partir da Política Nacional de Atenção Básica publicada pelas portarias ministeriais nos anos de 2011 e 2017.

\section{Materiais e Métodos}

Trata-se da análise documental de duas portarias 
ministeriais, que dispõem sobre a Política Nacional de Atenção Básica, publicadas em 2012 e 2017, respectivamente. Adotou-se a análise de conteúdo para identificar os elementos comuns e os conflitantes na redação das mesmas. Os documentos consultados são de domínio público e encontram-se disponíveis no site do Ministério da Saúde, portanto, não houve a necessidade de submissão do estudo no Conselho de Ética em Pesquisa (CEP) com seres humanos.

\section{Resultados}

As mudanças encontradas foram categorizadas conforme a tabela 1, abaixo:

Tabela 1. Categorias do estudo, segundo a análise documental.

\begin{tabular}{lllll}
\hline Categorias & PNAB 2012 & PNAB 2017 & \\
\hline Tipos de equipe de trabalho & ESF - ESB & $\begin{array}{l}\text { ESF, EAB } \\
\text { (opcional) }\end{array}$ & ESF \\
\hline Atribuições & $\begin{array}{l}\text { Atribuições próprias de } \\
\text { cada } \\
\text { profissional }\end{array}$ & $\begin{array}{l}\text { Incorporação de } \\
\text { atribuições } \\
\text { categorias }\end{array}$ & $\begin{array}{l}\text { novas } \\
\text { essas }\end{array}$ \\
\hline $\begin{array}{l}\text { Flexibilização da CH de } \\
\text { trabalho dos membros das } \\
\text { equipes }\end{array}$ & $\begin{array}{l}\text { CH de todos os membros } \\
=40 \mathrm{~h}\end{array}$ & $\begin{array}{l}\text { CH total da equipe }=40 \mathrm{~h} \\
\text { Categorias profissionais }\end{array}$ & $\begin{array}{l}\text { Médico, enfermeiro, } \\
\text { técnico de enfermagem, } \\
\text { ACS }\end{array}$ & $\begin{array}{l}\text { Médico, enico de enfermagem, } \\
\text { ACS, gerente da unidade }\end{array}$ \\
\hline Financiamento da ABS & $\begin{array}{l}\text { Blocos financeiros de } \\
\text { custeio específicos }\end{array}$ & $\begin{array}{l}\text { Único bloco financeiro de } \\
\text { custeio }\end{array}$ \\
\hline Apoio matricial & NASF & $\begin{array}{l}\text { NASF-AB } \\
\text { financiamento próprio) }\end{array}$ \\
\hline
\end{tabular}

Fonte: Os autores.

A primeira mudança percebida na PNAB de 2017 refere-se aos tipos de equipes que passam a compor a APS no Brasil: eSF (Estratégia de saúde da família), eAB (Equipe de atenção básica), eSB (Equipe de saúde bucal), NASF - AB (Núcleo de apoio à saúde da família - Atenção básica) e EACS (Estratégia de agentes comunitários de saúde) ${ }^{3}$, diferente da PNAB de 2011, que orientava a APS preferencialmente pela $\mathrm{ESF}^{4}$.

A PNAB de 2017 trouxe mudanças muito significativas para organização da APS e com possíveis efeitos sobre a integralidade do sistema de saúde, que antes considerava a ESF como opção preferencial de cuidado, com equipes completas e carga horária de trabalho compatível com a longitudinalidade, e passou a aceitar outras formas de equipes de trabalho, com o número de profissionais e suas cargas horárias de trabalho reduzidos ${ }^{3}$.

A equipe aceita antes da publicação de 2017 era a eSF, que era composta obrigatoriamente por médico, enfermeiro, auxiliar em enfermagem, agentes comunitários de saúde, e depois, por portaria específica, por dentista e auxiliar em saúde bucal ${ }^{4}$. Atualmente, com a nova portaria em vigor, aceita-se equipes com menos profissionais para áreas sem vulnerabilidades ${ }^{3}$. São as chamadas Equipes de atenção básica (eAB), que ficarão responsáveis por populações compostas por 2000 a 3500 pessoas $^{3}$. Essas equipes são compostas obrigatoriamente por médico, enfermeiro e técnico em enfermagem, podendo agregar dentista e auxiliar de saúde bucal ${ }^{3}$. São aceitas, porém não obrigatórias, outras equipes na composição das eAB, tais como, a equipe de saúde bucal (eSB), que pode ser composta por um dentista e um auxiliar em saúde bucal ou técnico em saúde bucal, ou ainda, por um dentista, e dois técnicos em saúde bucal ${ }^{3}$. Essa eSB pode compor a eSF ou a $\mathrm{e} \mathrm{AB}$, conforme a estrutura estabelecida para cada localidade ${ }^{3}$.

Ao cirurgião dentista integrante da eSF ou eAB, foi acrescentada a função de elaborar riscos e planos de cuidados para pacientes crônicos 5 . A nova modalidade de equipe aceita $(\mathrm{e} A B)$ possui distribuição flexível da carga horaria dos profissionais ${ }^{3}$, com a carga horária mínima de 10 horas, podendo haver no máximo três profissionais em mesma categoria ${ }^{3 ; 5}$.

Outra equipe aceita é a Estratégia de agentes comunitários de saúde (EACS), que é composta por agentes comunitários de saúde (ACS) e um enfermeiro supervisor ${ }^{3}$. Cada ACS deve ser responsável por uma área de no máximo 750 pessoas ${ }^{3}$. O planejamento das atividades deve ser de acordo com as necessidades locais, sempre priorizando a população com mais risco e vulnerabilidade ${ }^{3,4}$. A atuação das EACS deve visar integralidade entre a equipe e entre a equipe e paciente. Além disso, os ACS se tornaram responsáveis por aferição de pressão arterial e de hemoglicemia da população local (capacitação é oferecida) ${ }^{3}$.

Recomenda-se que as UBS tenham seu funcionamento em torno de 40 horas semanais, minimamente 5 dias por semana durante 12 meses4. Entretanto, com a chegada da PNAB de 2017, permitiuse a flexibilização da carga horária de trabalho de seus profissionais ${ }^{3}$, podendo inclusive superar a carga horária integral de trabalho proposta na publicação de 2011 em alguns casos. Em relação a eSF, ainda há a obrigatoriedade de carga horária de 40 horas semanais para todos os profissionais de saúde da equipe, porém, sem que haja a necessidade de sua existência na APS atual $^{3}$.

A PNAB publicada em 2017 estabeleceu uma carga horária mínima por profissional nas equipes de atenção básica de 10 horas semanais, com no máximo 3 profissionais por categoria, devendo somar 40 horas semanais para o grupo/equipe, e não por profissional, como anteriormente prevista $^{3}$. Vale ressaltar que na portaria de 2011, era previsto que o profissional poderia ter 8 horas semanais de dedicação a atividades de educação e formação, o que não aparece na portaria de 2017.

Como suporte matricial para as eSF, a PNAB de 2012 propunha o funcionamento dos Núcleos de Apoio 
à Saúde da Família (NASF), que passaram a se chamar na PNAB de 2017, de núcleos ampliados em saúde da família e atenção básica (NASF - AB), com incentivo muito menor para o seu funcionamento. Os NASF foram inicialmente criados pelo Ministério da Saúde, em 2008, com o objetivo de apoiar a consolidação da Atenção Primária no Brasil, ampliando as ofertas de saúde na rede de serviços, assim como a resolutividade, a abrangência e o alvo das ações, e passaram a se chamar NASF-AB ${ }^{3}$ para contemplar o suporte matricial, ainda presente, da nova modalidade de equipe de $\mathrm{AB}$, as e $\mathrm{AB}^{3}$.

Esta atuação do NASF-AB integrada às eSF e às $\mathrm{e} A \mathrm{~B}$ permite realizar discussões de casos clínicos, possibilita $o$ atendimento compartilhado entre profissionais, tanto na Unidade de Saúde, como nas visitas domiciliares; permite a construção conjunta de projetos terapêuticos de forma que amplia e qualifica as intervenções no território e na saúde de grupos populacionais. Essas ações de saúde também podem ser intersetoriais, com foco prioritário nas ações de prevenção e promoção da saúde. Qualquer município que possua eSF pode ter $\mathrm{NASF}^{4}$. O NASF 1 abrange de 5 a 9 eSFs. O NASF 2 abrange de 3 a 4 eSFs. O NASF 3 pode se estabelecer em qualquer município, desde que tenha $\mathrm{eSF}^{6}$. Com a mudança de nome para $\mathrm{NASF}-\mathrm{AB}$, as equipes que atuam na atenção básica que regulamentarão as agendas ${ }^{3,4}$.

Após a implementação da PNAB 2012, houve aumento da carga de responsabilidade dos municípios no financiamento do SUS ao longo dos anos e, a baixa participação do ente estadual ${ }^{1}$. Além disso, os gestores municipais reivindicaram mais independência em questão de financiamento para o município. Contudo, dados apontam que os municípios carregam a maior parte do custeio das equipes de saúde ${ }^{1}$.

Houve também uma mudança no protocolo de financiamento federal do SUS. Até então, o financiamento era repassado para 6 blocos, sendo 5 deles de custeio e um de investimento6. São eles: bloco de média e alta complexidade hospitalar e ambulatorial, Bloco de atenção básica, bloco de vigilância em saúde, bloco de assistência farmacêutica, bloco de gestão do SUS e o bloco de investimento6. O bloco de investimento é dedicado a ampliação e expansão das unidades e projetos de saúde6. Com a PNAB 2017, houve a unificação dos 5 blocos de custeio em 1 e o bloco de investimento foi mantido, portanto, temos atualmente 2 grandes blocos ${ }^{6}$.

Em 2016, a aprovação da proposta de emenda constitucional $\mathrm{n}^{\circ}$ 241/2016 que propunha um teto de gastos precedeu a portaria 2.436 de 2017. A PEC criou um congelamento nos gastos do governo com saúde e educação por 20 anos, fazendo com que os financiamentos pudessem sofrer alteração em relação a inflação. A proposta visava equilibrar os cofres públicos. O então ministro da saúde, Ricardo Barros, apoiou a criação de planos populares privados de saúde e a desregulamentação do setor suplementar ${ }^{1}$.

A Agência Nacional de Saúde Suplementar (ANS) é o setor que regula as atividades das operadoras de planos de saúde privados no Brasil, estabelecendo normativas para satisfação dos usuários e prestadores de serviço ${ }^{7}$. Atualmente, a ANS vem estimulando o setor privado a praticar programas de promoção de saúde, tendo como base o método do setor público de atenção primaria ${ }^{7}$. As medidas tomadas pelo governo como a PEC do teto e a flexibilização das equipes de saúde causarão um enfraquecimento drástico na saúde pública, e fortalecerá a iniciativa privada no setor?

Houve a introdução de um novo profissional na equipe, o gerente de atenção básica ${ }^{3}$, categoria profissional inexistente na proposta da PNAB publicada em 2011. Esse novo profissional não necessita ter formação na área ${ }^{3}$ e sua função é apenas gerenciar o funcionamento e os atendimentos, visando liberar o enfermeiro da unidade, que antes administrava a unidade de saúde, para cuidar de funções não administrativas, como preventivos, pré-natal, e grupos operativos ${ }^{3}$.

A partir da portaria em vigor publicada em 2017, os usuários do SUS poderão buscar atendimento em qualquer unidade básica do município, com pontos eletrônicos universalizados ${ }^{3}$, ou seja, o sistema de prontuários e atendimentos estará completamente integrado num banco de dados que poderá ser acessado de qualquer unidade básica de saúde ${ }^{3}$. Sem a chamada "regionalização", o usuário poderá ser atendido em um local de sua preferência, podendo ser atendido na unidade mais próxima de onde exerce suas atividades. Isso prejudicará o vínculo da equipe com os pacientes, e consequentemente a longevidade do atendimento como um todo.

Agora, com a publicação de 2017 em vigor, atribui-se ao gestor municipal autonomia para definição de prioridades e diretrizes locais ${ }^{3}$, através de uma margem financeira de manobra, para agir de acordo com as necessidades da população de seu território ${ }^{3}$. Ainda no mesmo artigo, fica mantida a obrigação do município em atualizar os serviços, carga horária, equipamentos e cadastros das equipes no o Sistema de cadastro nacional de estabelecimentos de saúde (SCNES) ${ }^{3}$. Tal autonomia se mostra importante, tendo em vista que as populações de diferentes territórios possuem algumas necessidades em específico. A atualização do SCNES é muito importante para controle das condições das equipes e dos serviços prestados. Tudo deve ser devidamente registrado para que não haja falta de recursos financeiros pela falta de alimentação de dados. 


\section{Discussão}

Dentre as várias mudanças que a nova PNAB de 2017 impõe, a previsão de funcionamento de outros tipos de equipes de saúde, além das tradicionais EqSF, afetará o controle de doenças nas diferentes regiões brasileiras. Com a ausência dos agentes comunitários de saúde (ACS), as visitas domiciliares poderão ser extintas, acometendo diretamente os levantamentos de dados epidemiológicos, a prevenção e a instrução da comunidade. Além de não contribuir para o vínculo entre a equipe e pacientes, essa mudança gerará um cuidado mais superficial em saúde.

Ao se aceitar a Equipe de Atenção Básica (EAB), aceita-se também o retrocesso no cuidado em saúde, pois a atenção à saúde deixa de ser longitudinal (contínua) e prestada por uma equipe multiprofissional, e passa a ser prestada por categorias profissionais isoladas, diferente do que propunha a PNAB publicada em 2011, sem a obrigatoriedade de se ter mais todos os profissionais durante a semana toda na unidade ${ }^{3} . \mathrm{Na}$ $\mathrm{EAB}$, há uma previsão de cumprimento de 40 horas semanais para a equipe como um todo ${ }^{3}$, mas não com obrigação de 40 horas para todos os membros da equipe, ou seja, o que antes eram 40 para todos (médicos, CD, enfermeiros etc.) passa a ser a $\mathrm{CH}$ total ${ }^{3}$, podendo ter um médico com apenas 10 horas de trabalho na equipe, desde que a unidade funcione 40 horas semanais ${ }^{3}$. Essa mudança compromete a integralidade da atenção à saúde e a universidade do acesso, pela falta ausência do profissional na unidade.

A criação de equipes com dedicação parcial à Atenção Primária e a readequação da equipe profissional com menor carga horária no Serviço tem sido consideravelmente discutida e criticada devido à possibilidade de fragmentação do processo de trabalho com múltiplos vínculos profissionais e menor vínculo entre a população e a equipe de referência ${ }^{5}$.

Além disso, a integralidade é novamente afetada quando o documento de 2017 permite flexibilizar a existência da Equipe de Saúde Bucal (ESB) na unidade de atenção básica, prescindindo da sua presença se assim o desejar. A ESB passa a compor a chamada equipe ampliada de saúde ${ }^{3}$, não prevista para todas as realidades brasileiras.

Acredita-se que as diferentes áreas da saúde são interligadas e devem trabalhar visando a integralidade nas suas ações para com o paciente. Muitas alterações são detectadas por manifestações orais específicas, contribuindo para o diagnóstico das mais diversas doenças. Aliás, os problemas odontológicos estão inclusos nos problemas de saúde pública, pois têm alta prevalência e causa grande impacto nas pessoas, devido aos desconfortos, dores e problemas estéticos que influenciam na vida social do indivíduo ${ }^{8}$.
Outra mudança promovida pela nova PNAB de 2017 refere-se às funções exercidas pelos membros das equipes de trabalho. Ao cirurgião dentista integrante da EqSF ou EqAB, foi acrescentada a função de elaborar riscos e planos de cuidados para pacientes crônicos ${ }^{3}$, embora a PNAB de 2012 já apontasse a necessidade do cuidado longitudinal dos usuários, característica necessária para aqueles que dependem de terapêutica longa, como portadores de doenças crônicas. No entanto, a PNAB de 2017 específica e instrui a elaboração dos planos terapêuticos singulares para esses usuários.

Já os ACS tornaram-se responsáveis por aferição de pressão arterial e de hemoglicemia da população local $^{3}$, embora capacitação a eles seja oferecida, na PNAB de 2017, assumindo assim, atribuições até então, exclusivas de outros profissionais ${ }^{3}$, no sentido de garantir o cuidado a quem precisa. Porém, cabe lembrar que a formação exigida desses profissionais é o ensino fundamental, o que levanta o debate sobre a existência ou não das habilidades e competências necessárias para tal exercício, que depende de cognição apropriada.

Ainda em relação ao processo de trabalho, a criação do cargo de gerente de unidade de saúde e a possibilidade de sua ocupação por um profissional sem formação na área ${ }^{3}$, pode ser extremamente prejudicial, justamente pela falta de conhecimento técnico específico e habilidade de gestão, considerando os inúmeros cursos de formação na área capazes de instrumentalizar a sua atuação. Essa função vinha sendo desempenhada pelo enfermeiro, que acumulava durante a sua formação a competência para coordenação e comando das equipes, integrando administração e os protocolos de atendimento. A figura do gerente da unidade de saúde a princípio favorece a organização do serviço na APS, entretanto, sem a necessidade de formação na área, diminui a qualidade do serviço.

No novo desenho da regionalização, com PNAB de 2017, os usuários das unidades de saúde poderão buscar atendimento em qualquer unidade básica do município, sem compromisso com o território definido para sua consulta ${ }^{3}$, prevalecendo a sua vontade em detrimento da capacidade do sistema de saúde e/ou a lógica dos encaminhamentos de forma racional e equânime. Por mais que o novo desenho tem o objetivo de facilitar o acesso do usuário, o pretendido vínculo entre profissional e paciente estará prejudicado, a longevidade do acompanhamento extremamente desfavorecida. Além do mais, a territorialização é importante para que os profissionais atendam e planejem os projetos, atividades e registros, considerando as especificidades de cada território ${ }^{4}$.

E por fim, a Portaria GM n 2436 altera as normas de financiamento estatal, fundindo em um bloco único todas as receitas de custeio dos municípios6. Tal mudança ocorreu sob a premissa de que as movimentações financeiras seriam mais rápidas 
e ocorreria uma agilização no processo. Porém, essa nova regra atribui poder de remanejamento de rubricas excessivo ao governante local. Antes, os recursos eram predestinados, com rubricas pré-definidas e só podendo ser utilizados nas áreas para as quais eram destinadas6. A PNAB 2017 possibilitou ao governante direcionar os recursos para custeio de maneira arbitraria. Desta forma, alguns setores da saúde, como a própria Atenção Básica à Saúde (ABS), poderão ser extremamente prejudicadas pelo subfinanciamento, quando o gestor desvincular parte da sua receita para áreas de maior interesse e/ou visibilidade política.

Para aumentar ainda mais o subfinanciamento do setor saúde, a Emenda Constitucional 241/2016 propôs um novo Regime Fiscal com limite dos gastos federais, com exceção dos juros da dívida pública. Nessa nova regra, implica-se um congelamento das despesas do Governo Federal, que visa o corte de gastos públicos. Os gastos ficarão estagnados tendo como referência o ano de 2017, recebendo apenas correções de acordo com as alterações inflacionárias ${ }^{9}$.

A possibilidade de aumentos nos gastos para saúde existe, de fato, porém deve ser feita somente a partir da redução de gastos primordiais, nos quais o setor saúde não se enquadra, causando grande impacto no setor social e na diminuição do Estado com protetor social ${ }^{9}$.

Ao invés dos gastos serem ampliados com as UBS, eles serão estagnados e reduzidos escalonadamente. A criação de novas equipes como as $\mathrm{eAB}$, com menor número de profissionais é uma evidência das intenções de cortes de recursos para a saúde ${ }^{9}$. Os favorecidos serão os segmentos de saúde privada, que já são estimulados pela ANS a adotarem programas de promoção de saúde, baseando-se justamente no modelo público já adotado?

\section{Considerações Finais}

Algumas das principais mudanças demonstradas na análise documental foram: em relação a tipos de equipes admitidas, atribuições, carga horária de membros das equipes, categorias profissionais, financiamento da ABS e apoio matricial.

Com a implementação da PNAB 2017, são admitidas as chamadas $\mathrm{eAB}$, que contam com menor número de profissionais na equipe e consequentemente, menor vínculo e participação na comunidade. A equipe de saúde Bucal passa a ser opcional.

As atribuições eram próprias de cada categoria profissional, mas com a PNAB 2017, houve incorporação de novas atribuições as categorias.

A carga horária de trabalho estipulada era de 40 horas semanais cumpridas por todos os membros da equipe. A PNAB 2017 exige que a carga horária total da equipe seja de 40 horas, podendo haver mais profissionais dividindo a carga horária total. Houve uma drástica flexibilização.

A PNAB 2017 admite uma nova categoria profissional chamada gerente da unidade, que não necessita ter formação na área da saúde. A função era responsabilidade do enfermeiro da unidade, que desempenhava o gerenciamento da UBS.

$\mathrm{O}$ financiamento da $\mathrm{ABS}$, que eram em blocos financeiros específicos de custeios, passou a serem vistos apenas como um único bloco.

$\mathrm{O}$ apoio matricial foi comprometido com a mudança do financiamento da ABS. O NASF passou a se chamar NASF-AB, e não possui mais financiamento próprio.

\section{Referências}

1. Melo EA, Mendonça MHM, Oliveira JR, Andrade GCL. Mudanças na Política Nacional de Atenção Básica: entre retrocessos e desafios. Saúde Debate 2018; 42(1):38-51.

2. Viana ALD, Dal Poz MR. A Reforma do Sistema de Saúde no Brasil e o Programa de Saúde da Família. Rev. Saúde Coletiva 2005;15:225- 264.

3. Brasil. Ministerio da Saúde. Portaria GM no 2436 de 21 de setembro de 2017. Aprova a Política Nacional de Atenção Básica, estabelecendo a revisão de diretrizes para a organização da Atenção Básica, no âmbito do Sistema Único de Saúde (SUS). 2017.

4. Brasil. Ministerio da Saúde. Portaria GM n 2488 de 21 de outubro de 2011. Aprova a Política Nacional de Atenção Básica, estabelecendo a revisão de diretrizes para a organização da Atenção Básica, no âmbito do Sistema Único de Saúde (SUS). 2011.

5. Ferreira JS, Ladeia LEG. As Implicações da Política Nacional de Atenção Básica (PNAB) na Dinâmica dos Serviços de Saúde. Rev. Mult. Psic. 2018;12(42):681-695.

6. Confederação Nacional de Municípios - CNM. Mudanças no Financiamento da Saúde; 2018; Brasília.

7. Barbosa ML, Celino SDM, Costa GMC. A Estratégia de Saúde da Família no setor suplementar: a adoção do modelo de atenção primária na empresa de autogestão. Rev Interface 2015;19(55):1101-8.

8. Araújo LC. O Programa da Família pelo olhar do cirurgião-dentista. Rio Grande do Norte. Tese de Mestrado em Programa de Saúde Familiar UFRN; 2005.

9. Rossi P, Dweck E. Impactos do Novo Regime Fiscal na saúde e educação. Cad. Saúde Pública 2016; 32(12):e0019431. 